\title{
Correlation of MRI-derived adipose tissue measurements and anthropometric markers with prevalent hypertension in the community
}

\author{
Roberto Lorbeer ${ }^{a, f}$, Susanne Rospleszcz ${ }^{b}$, Christopher L. Schlett ${ }^{c}$, Sophia D. Heber ${ }^{g, i}$, \\ Jürgen Machann ${ }^{e, g_{, j}, \text { Barbara Thorand }}{ }^{b}$, Christa Meisinger ${ }^{b, k}$, Margit Heier ${ }^{b, d}$, Annette Peters ${ }^{b, e, f}$, \\ Fabian Bamberg ${ }^{\mathrm{a}, \mathrm{g}}$, and Wolfgang Lieb ${ }^{\mathrm{h}}$
}

\begin{abstract}
Objectives: To compare the correlations of MRI-derived adipose tissue measurements and anthropometric markers, respectively, with prevalent hypertension in a communitybased sample, free of clinical cardiovascular disease.
\end{abstract}

Methods: MRI-derived adipose tissue measurements were obtained in 345 participants (143 women; age 39-73 years) of the KORA FF4 survey from Southern Germany using a 3-Tesla machine and included total adipose tissue (TAT), visceral adipose tissue (VAT), subcutaneous adipose tissue (SCAT), hepatic fat fraction (HFF), pancreatic fat fraction (PFF) as well as pericardial adipose tissue (PAT). In addition, the anthropometric markers body mass index, waist circumference, hip circumference, waist-hip ratio (WHR) and waist-height ratio (WHtR) as well as blood pressure measurements were obtained.

Results: The prevalence of hypertension was 33.6\% (women: $28 \%$, men: $38 \%$ ). VAT and PAT had the highest area under the curve (AUC) values for identifying individuals with prevalent hypertension (AUC: 0.75; 0.73, respectively), whereas $\mathrm{WH} R$ and waist circumference were best performing anthropometric markers (AUC: 0.72; 0.70, respectively). A 1SD increment of TAT was associated with the highest odd for hypertension in the age-adjusted and sex-adjusted model $(\mathrm{OR}=2.20,95 \% \mathrm{Cl} 1.67-2.91$, $P<0.001)$ and in the fully adjusted model $(O R=1.97$, $95 \% \mathrm{Cl} 1.45-2.66, P<0.001)$. TAT was the only MRIderived adipose tissue measurement that was associated with hypertension independently of the best performing anthropometric marker waist circumference in the fully adjusted model $(\mathrm{OR}=1.93,95 \% \mathrm{Cl} 1.00-3.72, P=0.049)$.

Conclusion: MRI-derived adipose tissue measurements perform similarly in identifying prevalent hypertension compared with anthropometric markers. Especially, TAT, VAT and PAT as well as WHtR and waist circumference were highly correlated with prevalent hypertension.

Keywords: adipose tissue, anthropometry, hypertension, MRI, population

Abbreviations: AUC, area under the curve; BIA, bioelectrical impedance analysis; $B P$, blood pressure; CVD, cardiovascular disease; HFF, hepatic fat fraction; OR, odds ratio; PAT, pericardial adipose tissue; PFF, pancreatic fat fraction; SCAT, subcutaneous adipose tissue; TAT, total adipose tissue; VAT, visceral adipose tissue; WHR, waisthip ratio; WHtR, waist-height ratio

\section{INTRODUGTION}

$\mathbb{1}$ levated blood pressure is a major cardiovascular risk factor that is considered a cardiovascular disease (CVD) equivalent [1]. On a parallel note, adiposity predisposes to cardiometabolic disease conditions [2,3], and hypertension is an important link between increased body fat distribution and cardiovascular outcomes [4,5].

It is not well established, however, which is the best adiposity measurement for cardiovascular risk assessment and which most strongly correlates with hypertension. As they are easy and cost-effective to measure, anthropometric markers have been investigated in a large number of studies, and are part of nonlaboratory-based prediction algorithms for CVD in primary care [6].

As anthropometry provides only indirect measurements of body fat distribution, it is of major interest to identify more accurate and direct measures of body fat and to explore their relation to CVD risk factors, including blood pressure. Bioelectrical impedance analysis (BIA) enables to distinguish between body fat mass and body fat-free mass.

Journal of Hypertension 2018, 36:1555-1562

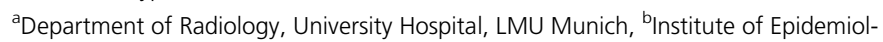
ogy II, Helmholtz Zentrum München, Neuherberg, 'Department of Diagnostic and Interventional Radiology, University Hospital Heidelberg, dKORA Myocardial Infarction Registry, Central Hospital of Augsburg, Augsburg, e'German Center for Diabetes Research (DZD e.V.), Neuherberg, ${ }^{\dagger}$ German Center for Cardiovascular Disease Research (DZHK e.V.), Munich, ${ }^{9}$ Department of Diagnostic and Interventional Radiology, Eberhard Karl University Tübingen, Tübingen, Institute of Epidemiology, Christian Albrecht University, Kiel, Germany, 'CTMH Doctors Hospital, George Town, Grand Cayman, Cayman Islands, 'Institute for Diabetes Research and Metabolic Diseases (IDM), Helmholtz Centre Tübingen and ${ }^{k}$ Chair of Epidemiology, LMU Munich, UNIKA-T Augsburg, Germany

Correspondence to Roberto Lorbeer, PhD, Department of Radiology, Ludwig Maximilian University Hospital, Pettenkoferstr. 8a, 80336 München, Germany. Tel: +49 89 4400 59289; fax: +49 894400 59282:

e-mail: roberto.lorbeer@med.uni-muenchen.de

Received 1 December 2017 Revised 28 February 2018 Accepted 1 March 2018 J Hypertens 36:1555-1562 Copyright (C) 2018 Wolters Kluwer Health, Inc. All rights reserved.

DOI:10.1097/HJH.0000000000001741 
However, in a Chinese community-based sample, BIAderived body fat displayed weaker associations with various metabolic abnormalities and hypertension as compared with anthropometric markers [7].

By contrast, different imaging technologies, including computed tomography (CT) and MRI, allow the visualization and quantification of direct measures of body and organ adipose tissue. Thus far, particularly, little is known about the association of MRI-derived adipose tissue measurements with hypertension compared with established anthropometric markers. MRI measures of body and organ fat obtained in our sample include total adipose tissue (TAT), visceral adipose tissue (VAT), subcutaneous adipose tissue (SCAT), hepatic fat fraction (HFF), pancreatic fat fraction (PFF) as well as pericardial adipose tissue (PAT). In the present analysis, we aimed to compare the associations of these MRI-derived adipose tissue measurements on the one hand and of anthropometric markers on the other hand with prevalent hypertension. Specifically, we assessed the performance of these different adiposity measures in identifying people with prevalent hypertension in a sample from the general population, without clinical CVD.

\section{METHODS}

\section{Study sample}

The KORA FF 4 study is the second follow-up examination of the KORA S4 study ('Cooperative Health Research in the Region of Augsburg'), a population-based health survey conducted in the city of Augsburg (Southern Germany) and two surrounding counties between 1999 and 2001. Of all 4261 participants of the KORA S4 baseline study, 2279 participants also participated in the 14-year follow-up FF4 study conducted between 2013 and 2014. In a FF4 MRI substudy, a total of $400 \mathrm{FF} 4$ participants without history of stroke, myocardial infarction and arterial vessel occlusion were examined by MRI [8]. Participants with missing data for TAT $(n=16)$, HFF $(n=11)$, PFF $(n=4)$ and PAT $(n=24)$ were excluded from the present analysis, yielding an analytical sample of 345 participants ( 143 women; age $39-73$ years).

The investigations were carried out in accordance with the Declaration of Helsinki, and written informed consent was provided by all participants. The study was approved by the ethics committee of the Bavarian Chamber of Physicians, Munich (S4: EC No. 99186 and for genetic epidemiological questions 05004, F4 and FF4: EC No. 06068). The MRI examination protocol was approved by the ethics committee of the Ludwig Maximilian University Hospital, Munich.

\section{Magnetic resonance examination and MRI-derived fat measurements}

MR examinations were performed on a 3-Tesla Magnetom Skyra (Siemens AG, Healthcare Sector, Erlangen, Germany) using an 18-channel body array coil in combination with the table-mounted spine matrix coil [8]. Participants were scanned in a supine position.

\section{Total adipose tissue, visceral adipose tissue and subcutaneous adipose tissue}

On the basis of the volume-interpolated 3D in/opposedphase VIBE-Dixon sequence, a fat selective tomogram was calculated (slice thickness $5 \mathrm{~mm}$ at $5 \mathrm{~mm}$ increment). An inhouse algorithm based on Matlab R2013a was used to automatically quantify the TAT from the femoral head to the cardiac apex, VAT from the femoral head to the diaphragm and SCAT from the femoral head to the cardiac apex. All segmentations were manually adjusted if necessary. TAT, VAT and SCAT were indexed by squared height $\left(\mathrm{m}^{2}\right)$.

\section{Hepatic fat fraction}

The multiecho Dixon was based on a Volume Interpolated Body Examination (VIBE) sequence with the following parameters: TR $8.90 \mathrm{~ms}$, six echos with an increment of $1.23-7.38 \mathrm{~ms}$, flip angle $4^{\circ}$, matrix $256 \times 179$. Slice thickness was $4 \mathrm{~mm}$. For the estimation of liver proton density fat fraction, confounding effects of $\mathrm{T} 2 *$ decay and the spectral complexity of fat were taken into account [9]. Acquisition time was approximately $15 \mathrm{~s}$. Data was analyzed using Osirix (Vers. 4.1 64-bit, Pixmeo SARL, Bernex, Geneva, Switzerland). A region of interest was manually drawn on one slice at the height of the portal vein including the whole liver parenchyma avoiding large vessels and surrounding extrahepatic tissue to measure HFF at the level of the portal vein. Data are given in percentage (\%).

\section{Pancreatic fat fraction}

For quantitative assessment of pancreatic fat tissue content, one or two circular regions of interest (ROI) covering an area of approximately $100 \mathrm{~mm}^{2}$ were drawn into the pancreatic head (caput), the pancreatic body (corpus) and the pancreatic tail (cauda) on different MRI layers, using a dedicated off-line workstation (Syngo Via, Siemens Healthcare, Erlangen, Germany). Images with severe image artefacts (e.g. phase swaps) were excluded from the analysis. Data are indexed in percentage (\%).

\section{Pericardial adipose tissue}

Pericardial adipose tissue was defined as any mediastinal fat between the pulmonary artery bifurcation and the diaphragm, this includes fat inside the visceral layer of the pericardial sac in close proximity to the myocardium as well as outside of the pericardial sac [10]. Applying an automated procedure based on cluster analysis (Matlab R2013a) PAT was quantified between thoracic diaphragm and vascular bifurcation of the pulmonary artery and carefully avoiding inclusion of mediastinal adipose tissue.

\section{Anthropometric measurements}

Weight was measured with an electronic scale (SECA 635, SECA 877), standing height with a stadiometer (SECA 242) allowing accurate measurements up to $0.1 \mathrm{~kg}$ and $0.1 \mathrm{~cm}$, respectively. BMI was calculated as weight divided by squared height $\left(\mathrm{kg} / \mathrm{m}^{2}\right)$. Waist circumference and hip circumference were measured with an inelastic multicolored measuring tape (Fa Hoechstmass) in centimeters to the closest $0.1 \mathrm{~cm}$. Hip circumference was measured at the widest protrusion of the gluteal region between the superior border of the iliac crest and crotch. Waist circumference was measured at the level midway between the lower rib margin and the iliac crest whereas the participants breathed 
out gently. Waist circumference was divided by hip circumference to get waist-hip ratio (WHR) and by height to get waist-height ratio (WHtR).

\section{Blood pressure measurements and definition of hypertension}

SBP and DBP measurements were obtained three times at the right arm of seated participants after a minimum resting period of $5 \mathrm{~min}$. The time interval between readings was 3 min. An oscillometric digital BP monitor (HEM-705CP; Omron Corporation, Tokyo, Japan) was used and one of two cuff sizes was applied as appropriate for the participant's arm circumference. The mean of the second and third $\mathrm{BP}$ measurements was used for the present analyses [11]. Hypertension was defined as SBP at least $140 \mathrm{mmHg}$ or DBP at least $90 \mathrm{mmHg}$ [12] or use of antihypertensive medication under the awareness of having hypertension. Medication intake within the last 7 days was recorded during a medical interview by computer-based software, and participants were also asked to bring their medication packages with them. Antihypertensive medication was defined according to the German Hypertension Association and included antihypertensives, diuretics, beta blocking agents, calcium channel blockers or agents acting on the renin-angiotensin system [13]. If participants reported that they had ever been told by a physician to have high or elevated $\mathrm{BP}$, they were characterized as being aware of hypertension.

\section{Covariables}

A broad range of health-related variables were measured in KORA FF4 by standardized interview, a comprehensive physical examination and laboratory analyses. Participants were classified as never-smoker, ex-smoker or current smoker; and as being physically active if they did regular sports in summer or winter for at least $1 \mathrm{~h}$ per week or as physically inactive if they did irregular $1 \mathrm{~h}$ or less of sports per week [14]. Alcohol intake was assessed using a validated recall method, calculating alcohol intake in grams per day from participants' self-reported intake of beer, wine, sparkling wine or distilled spirits over the previous weekend and workday [15].

Diabetes was defined according to the WHO definition as a 2-h plasma glucose concentration measured by oral glucose tolerance test equal or above $200 \mathrm{mg} / \mathrm{dl}$ and/or a fasting glucose level above $125 \mathrm{mg} / \mathrm{dl}$ [16]. Laboratory measurements including triglycerides, total cholesterol, high-density and low-density lipoprotein cholesterol were described elsewhere [17].

\section{Statistical analyses}

Descriptive characteristics of normotensive and hypertensive participants are provided as median and interquartile range for continuous measurements and absolute numbers and percentage values for categorical measurements. Differences between normotensive and hypertensive participants were tested using either Mann-Whitney $U$ test (continuous data) or $\chi^{2}$-test (categorical data). Power analysis revealed that the sample size of the present study $(N=345)$ provides $80 \%$ power at a significance level of $\alpha=0.05$ to detect differences in TAT between the normotensive group $\left(N_{1}=229\right.$, mean $\left.=3.4, \mathrm{SD}=1.8\right)$ and the hypertensive group $\left(N_{2}=116\right)$ of $0.581 / \mathrm{m}^{2}$.

Receiver-operating characteristic (ROC) curves to distinguish individuals with prevalent hypertension from those without were estimated separately for each MRI-derived adipose tissue measurement and for each anthropometric marker. We performed age-adjusted and sex-adjusted, as well as multivariable-adjusted logistic regression models including age, diabetes mellitus, physical activity, smoking status, alcohol consumption, total cholesterol and HDLcholesterol. We performed multivariable adjusted models to estimate the association of each adiposity marker with prevalent hypertension independently of potentially confounding variables (including traditional cardiovascular risk factors). In sex-stratified analysis, multivariable-models were additionally adjusted for hormone replacement therapy in women. Likelihood-ratio tests were used to test the improvement of area under the curve (AUC) values between a basic model including only traditional risk factors vs. the basic model with an adiposity marker added. Differences of AUC values between different adiposity markers were evaluated by $\chi^{2}$-test.

Associations of adiposity traits (expressed as odds ratios per 1SD increment) with hypertension were evaluated by logistic regression models with age and sex-only adjustment (Model 1) and with multivariable adjustment using the covariates as mentioned above (Model 2). Odds ratios (ORs) for the association of each adiposity trait with hypertension were ordered from high to low and graphically displayed. In addition, the associations of MRI-derived adiposity traits with hypertension were tested upon additional adjustment for the most strongly associated anthropometric marker, waist circumference. In sensitivity analyses, the associations of MRI-derived adipose tissue measurements and of anthropometric traits with SBP, modeled as a continuous trait, were assessed by right censored normal regression, thereby accounting for antihypertensive treatment.

Effect modification by sex on the association between adiposity traits and hypertension was tested by including respective multiplicative interaction terms (adiposity trait $*$ sex) in the logistic regression model. In the analyses described above, all MRI-derived measurements were modeled as continuous traits. In an additional analysis, we evaluated how well different MRI-derived adiposity measurements and anthropometric markers can be used to differentiate between hypertensive participants and nonhypertensive participants, if the respective traits were dichotomized at the median (two groups for each marker with $50 \%$ in the group with values above the median and $50 \%$ below). For each adiposity marker, the prevalence of hypertension was compared between the two groups with values above vs. below the sex-specific median.

A $P$ value of less than 0.05 was considered statistically significant. Statistical analyses were performed using Stata 14.1 (Stata Corporation, College Station, Texas, USA).

\section{RESULTS}

Baseline characteristics of the study sample, stratified by hypertension status, are provided in Table 1 . Hypertension 
TABLE 1. Characteristics of the study sample $(N=345)$

\begin{tabular}{|c|c|c|c|}
\hline & $\begin{array}{l}\text { Without hypertension } \\
\qquad N=229\end{array}$ & $\begin{array}{l}\text { Hypertension } \\
\qquad N=116\end{array}$ & P value ${ }^{a}$ \\
\hline \multicolumn{4}{|l|}{ Covariates } \\
\hline Age (years) & $52(46 ; 61)$ & $62(56 ; 67)$ & $<0.001$ \\
\hline Males & $126(55.0 \%)$ & $76(65.5 \%)$ & 0.062 \\
\hline Smoking status & & & 0.130 \\
\hline Never-smoker & $86(37.6 \%)$ & $41(35.3 \%)$ & \\
\hline Ex-smoker & $92(40.2 \%)$ & $58(50.0 \%)$ & \\
\hline Current smoker & $51(22.3 \%)$ & $17(14.7 \%)$ & \\
\hline Alcohol consumption (g/day) & $8.6(0.9 ; 24.6)$ & $11.9(0.0 ; 38.6)$ & 0.191 \\
\hline Physically active & $146(63.8 \%)$ & $59(50.9 \%)$ & 0.021 \\
\hline Diabetes mellitus & $12(5.2 \%)$ & $33(28.5 \%)$ & $<0.001$ \\
\hline $\mathrm{HDL}-\mathrm{C}(\mathrm{mmol} / \mathrm{l})$ & $1.58(1.29 ; 1.91)$ & $1.51(1.21 ; 1.805)$ & 0.048 \\
\hline LDL-C (mmol/l) & $3.57(2.97 ; 4.16)$ & $3.46(3.01 ; 4.105)$ & 0.960 \\
\hline Total cholesterol (mmol/l) & $5.61(4.99 ; 6.25)$ & $5.48(4.91 ; 6.18)$ & 0.763 \\
\hline Triglycerides (mmol/l) & $1.17(0.83 ; 1.63)$ & $1.36(1.05 ; 2.055)$ & $<0.001$ \\
\hline Weight (kg) & $78.4(68.7 ; 90.4)$ & $85.1(76.9 ; 95.7)$ & $<0.001$ \\
\hline Height (m) & $1.73(1.64 ; 1.80)$ & $1.72(1.64 ; 1.78)$ & 0.359 \\
\hline \multicolumn{4}{|l|}{ Anthropometric markers } \\
\hline BMI $\left(\mathrm{kg} / \mathrm{m}^{2}\right)$ & $26.6(23.9 ; 29.2)$ & $29.0(26.5 ; 32.1)$ & $<0.001$ \\
\hline Waist circumference $(\mathrm{cm})$ & $95.7(84.6 ; 103.5)$ & $104.3(98.2 ; 111.3)$ & $<0.001$ \\
\hline Hip circumference $(\mathrm{cm})$ & $104.6(99.9 ; 109.1)$ & $108.4(102.4 ; 113.8)$ & $<0.001$ \\
\hline Waist-hip ratio, WHR & $0.91(0.85 ; 0.96)$ & $0.97(0.90 ; 1.02)$ & $<0.001$ \\
\hline Waist-height ratio, WHtR & $0.55(0.50 ; 0.60)$ & $0.61(0.56 ; 0.66)$ & $<0.001$ \\
\hline \multicolumn{4}{|l|}{ Adipose tissue (MRI) } \\
\hline Total adipose tissue, TAT $\left(1 / \mathrm{m}^{2}\right)$ & $3.36(2.55 ; 4.83)$ & $5.03(3.75 ; 6.49)$ & $<0.001$ \\
\hline Visceral adipose tissue, VAT $\left(1 / \mathrm{m}^{2}\right)$ & $1.08(0.67 ; 1.62)$ & $1.96(1.37 ; 2.45)$ & $<0.001$ \\
\hline Subcutaneous adipose tissue, SCAT $\left(1 / \mathrm{m}^{2}\right)$ & $2.16(1.70 ; 3.10)$ & $2.88(2.10 ; 4.23)$ & $<0.001$ \\
\hline Hepatic fat fraction, HFF (\%) & $3.84(2.24 ; 6.97)$ & $9.56(4.10 ; 20.38)$ & $<0.001$ \\
\hline Pancreatic fat fraction, PFF (\%) & $4.73(3.1 ; 7.33)$ & $6.95(4.03 ; 14.77)$ & $<0.001$ \\
\hline Pericardial adipose tissue, PAT (ml) & $92.0(61.5 ; 135.6)$ & $145.8(111.1 ; 206.0)$ & $<0.001$ \\
\hline \multicolumn{4}{|l|}{ Blood pressure } \\
\hline $\mathrm{SBP}(\mathrm{mmHg})$ & $116(107 ; 124)$ & $133(120 ; 143)$ & $<0.001$ \\
\hline $\mathrm{DBP}(\mathrm{mmHg})$ & $73(68 ; 79)$ & $80(73 ; 89)$ & $<0.001$ \\
\hline Use of antihypertensive medication & - & $84(72.4 \%)$ & - \\
\hline
\end{tabular}

Data are given as number (percentage) or median (25th and 75th percentile). HDL-C, high-density lipoprotein cholesterol; LDL-C, low-density lipoprotein cholesterol.

${ }^{a} P$ values are from Mann-Whitney $U$ test (continuous data) or chi-square test (categorical data).

prevalence in the overall sample was 33.6\% (women: 28\%, men: 38\%). Hypertensive participants were older, more often ex-smokers and less physically active in leisure time compared with normotensive participants. All median values of MRI-derived adipose tissue measurements and anthropometric markers were higher in participants with hypertension compared with participants without hypertension.

Among the MRI-derived measures, VAT and PAT had the highest AUC values for identifying individuals with prevalent hypertension (AUC: 0.75; 0.73, respectively), whereas WHtR and waist circumference were the best performing anthropometric markers $(0.72 ; 0.70$, respectively, Table 2 and Supplemental Figure 1, http://links.lww.com/HJH/ A923). PFF and hip circumference had lowest AUC values for hypertension $(0.65 ; 0.63$, respectively).

In multivariable-adjusted models, AUC values were highest for TAT and SCAT (AUC: 0.80, respectively, Table 2 ), even though all adiposity traits improved the AUC beyond the basic model (AUC: 0.767), which included only the traditional risk factors (all $P \leq 0.016$ for improvement in AUC when added to a model with only traditional risk factor). Only AUC values of single adiposity traits differed among each other $(P<0.001)$ whereas AUC values upon age and sex as well as multivariable adjustment did not $(P=0.871$ for MRI-derived adipose tissue measurements;
$P=0.219$ for anthropometric markers). Furthermore, formal statistical comparisons revealed that there were no statistically significant differences between any of the MRI-derived adipose tissue measurements and any of the anthropometric adiposity measures (comparing the respective AUCs) in identifying prevalent hypertension (Supplemental Table 1, http://links.lww.com/HJH/A923).

A 1SD increment of TAT was associated with the highest odd for hypertension in the age-adjusted and sex-adjusted model $(\mathrm{OR}=2.20,95 \%$ CI 1.67-2.91, $P<0.001)$ and in the fully adjusted model $(\mathrm{OR}=1.97$, 95\% CI 1.45-2.66, $P<0.001$; Fig. 1 and Supplemental Table 2, http://links. lww.com/HJH/A923). Also SCAT and VAT were highly associated with hypertension in both models (all $P<0.001)$. Among the different anthropometric marker, waist circumference displayed the highest odd for hypertension in the age-adjusted and sex-adjusted model (OR $=2.19$, 95\% CI 1.63-2.93, $P<0.001)$ and in the fully adjusted model (OR $=1.92,95 \%$ CI 1.39-2.67, $P<0.001)$. Similarly, TAT and waist circumference were the most strongly associated marker of their respective groups (MRI markers and anthropometric markers, respectively) in relation to continuously modeled SBP (Supplemental Table 3, http://links.lww.com/HJH/A923). In a secondary analysis, when the best performing anthropometric marker waist circumference was added to the 
TABLE 2. Area under the curve values for different statistical models investigating the presence of prevalent hypertension

\begin{tabular}{|c|c|c|c|c|}
\hline $\mathbf{N}=\mathbf{3 4 5}$ & $\begin{array}{c}\text { Hypertension AUC } \\
(95 \% \mathrm{Cl}) \text { of single factor }\end{array}$ & $\begin{array}{l}\text { Hypertension AUC }(95 \% \mathrm{Cl}) \\
\text { of single factor with age and sex }\end{array}$ & $\begin{array}{l}\text { Hypertension AUC }(95 \% \mathrm{Cl}) \\
\text { single factor with basic model }\end{array}$ & $P$ value $^{b}$ \\
\hline & & & $\mathrm{AUC}_{\text {basic }}=0.7665$ & \\
\hline TAT & $0.72(0.67 ; 0.78)$ & $0.79(0.73 ; 0.84)$ & $0.80(0.75 ; 0.85)$ & $<0.001$ \\
\hline VAT & $0.75(0.70 ; 0.81)$ & $0.78(0.73 ; 0.83)$ & $0.79(0.75 ; 0.84)$ & $<0.001$ \\
\hline SCAT & $0.66(0.60 ; 0.72)$ & $0.77(0.72 ; 0.83)$ & $0.80(0.75 ; 0.84)$ & $<0.001$ \\
\hline HFF & $0.72(0.67 ; 0.78)$ & $0.76(0.71 ; 0.82)$ & $0.79(0.74 ; 0.84)$ & 0.002 \\
\hline PFF & $0.65(0.59 ; 0.72)$ & $0.74(0.68 ; 0.79)$ & $0.78(0.73 ; 0.83)$ & 0.016 \\
\hline \multirow[t]{2}{*}{ PAT } & $0.73(0.67 ; 0.78)$ & $0.76(0.70 ; 0.81)$ & $0.79(0.74 ; 0.84)$ & 0.001 \\
\hline & $P<0.001$ & $P=0.152$ & $P=0.871$ & \\
\hline $\mathrm{BMI}$ & $0.68(0.62 ; 0.74)$ & $0.77(0.72 ; 0.82)$ & $0.79(0.74 ; 0.84)$ & $<0.001$ \\
\hline Waist circumference & $0.70(0.65 ; 0.76)$ & $0.78(0.72 ; 0.83)$ & $0.79(0.75 ; 0.84)$ & $<0.001$ \\
\hline Hip circumference & $0.63(0.57 ; 0.69)$ & $0.75(0.70 ; 0.81)$ & $0.79(0.74 ; 0.84)$ & $<0.001$ \\
\hline WHR & $0.70(0.64 ; 0.76)$ & $0.76(0.71 ; 0.82)$ & $0.78(0.73 ; 0.83)$ & 0.003 \\
\hline \multirow[t]{2}{*}{ WHtR } & $0.72(0.66 ; 0.78)$ & $0.78(0.72 ; 0.83)$ & $0.79(0.75 ; 0.84)$ & $<0.001$ \\
\hline & $P<0.001$ & $P=0.110$ & $P=0.219$ & \\
\hline
\end{tabular}

Models either included only traditional risk factors (AUC $C_{\text {basic }}$, only individual adiposity traits of interest, only individual adiposity traits of interest combined with age and sex, or each

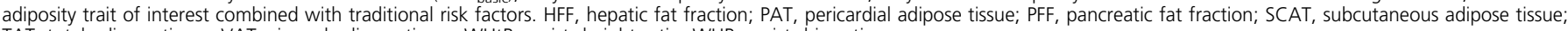
TAT, total adipose tissue; VAT, visceral adipose tissue; WHtR, waist-height ratio; WHR, waist-hip ratio.

a Basic risk factor model for hypertension includes: age, sex, diabetes, physical activity, smoking status, alcohol consumption, total cholesterol, HDL-cholesterol.

bikelihood-ratio test (comparison: basic model vs. basic risk factor model with adiposity risk factor).

multivariable-adjusted model with the different MRIderived adiposity measures, only TAT was associated independently with prevalent hypertension $(\mathrm{OR}=1.93,95 \% \mathrm{CI}$ $1.00-3.72, P=0.049$, Table 3 ). In order to further explore the association between total adipose tissue and hypertension, we provide different multivariable-adjusted models with an increasing number of potential confounders (Supplemental Table 4, http://links.lww.com/HJH/A923). In essence, the strength of association decreased slightly from the unadjusted model $(\mathrm{OR}=2.18[1.70 ; 2.81])$ to the fully adjusted model including waist circumference $(\mathrm{OR}=1.93$ [1.00; 3.72]).

Interaction analysis revealed an effect modification by sex regarding the association of PAT with hypertension
$(P=0.035)$. The association per 1SD increment in PAT with hypertension was stronger in women $(\mathrm{OR}=3.53,95 \% \mathrm{CI}$ $1.74-7.17, P<0.001)$ compared with men (OR $=1.36,95 \%$ CI $0.95-1.93, P=0.093$ ) in fully adjusted models (Fig. 2).

Whenever the different adiposity traits were dichotomized at the sex-specific median, the biggest difference in hypertension prevalence was observed between women with high PAT (45\% hypertension prevalence) and low PAT (11\% hypertension prevalence). In men, the biggest difference in hypertension prevalence was observed between men with high VAT (58\%) and low VAT (17\%) (Fig. 3). Interaction analysis revealed no further relevant adiposity marker that could significantly identify more individual hypertension risk in these subgroups. (a)

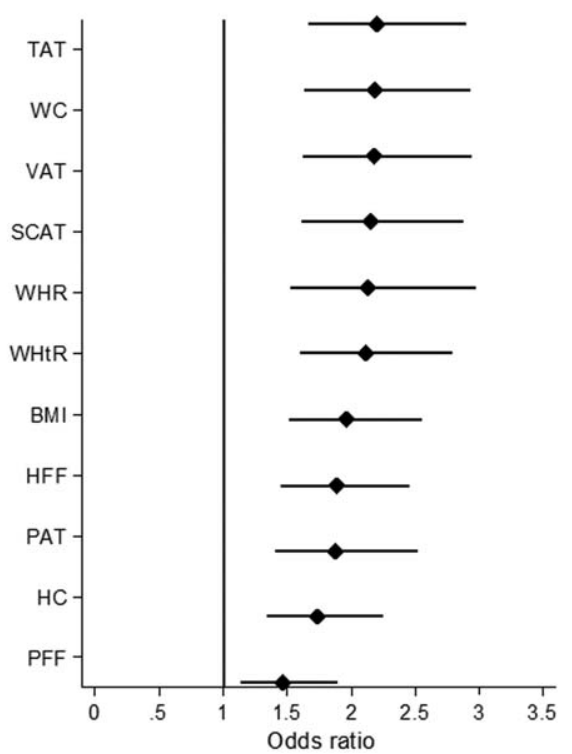

(b)

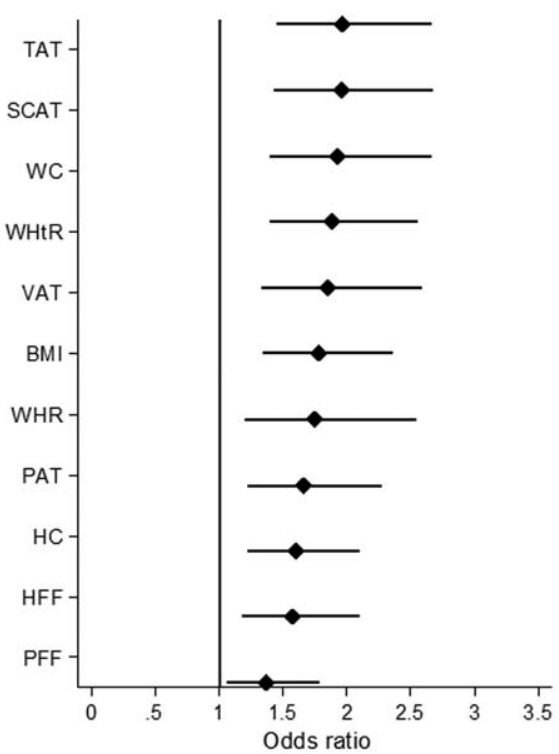

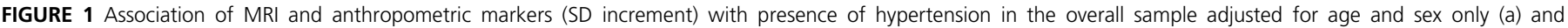
adjusted for age, sex, diabetes, physical activity, smoking status, alcohol consumption, total cholesterol and HDL-cholesterol (b) expressed by odds ratios and $95 \%$ confidence intervals. 
TABLE 3. Association of MRI-derived adiposity measurements with the presence of hypertension independent of cardiovascular risk factors and independent of the strongest associated anthropometric marker waist circumference

\begin{tabular}{lcc}
$\mathbf{N}=\mathbf{3 4 5}$ & Hypertension OR $(\mathbf{9 5} \% \mathbf{C l})$ & $\boldsymbol{P ~ v a l u e ~}^{\mathbf{a}}$ \\
TAT & $1.93(1.00-3.72)$ & 0.049 \\
VAT & $1.29(0.80-2.08)$ & 0.302 \\
SCAT & $1.68(0.92-3.06)$ & 0.090 \\
HFF & $1.28(0.94-1.76)$ & 0.120 \\
PFF & $1.17(0.89-1.55)$ & 0.250 \\
PAT & $1.27(0.88-1.83)$ & 0.193 \\
\hline
\end{tabular}

Data are odds ratios per SD increment of the respective adiposity measurements from logistic regression. HFF, hepatic fat fraction; PAT, pericardial adipose tissue; PFF, pancreatic fat fraction; SCAT, subcutaneous adipose tissue; TAT, total adipose tissue; VAT, visceral adipose tissue.

${ }^{a}$ Adjusted for age, sex, diabetes, physical activity, smoking status, alcohol consumption, total cholesterol, HDL-cholesterol, and waist circumference.

\section{DISGUSSION}

This is the first community-based study to investigate simultaneously the associations of MRI-derived adipose tissue measurements on the one hand and of established anthropometric markers on the other hand with prevalent hypertension. We conducted our analyses in a populationbased sample free of clinical cardiovascular diseases. The main observations can be summarized as follows. First, in general, MRI-derived adipose tissue measurements and anthropometric markers were both associated with hypertension in a relatively similar fashion. Second, whereas TAT, VAT and PAT were the best MRI-derived adipose tissue measurements to distinguish those with from those without hypertension, waist circumference and WHtR were the best performing anthropometric markers for identifying prevalent hypertension. Third, in women, the association between PAT and hypertension was stronger than in men.

\section{Association of anthropometric markers with hypertension}

Anthropometric markers are commonly used as surrogate markers of body fat distribution and obesity in prediction models for CVD and hypertension as they are relatively easy to measure $[18,19]$. In our sample, waist circumference and

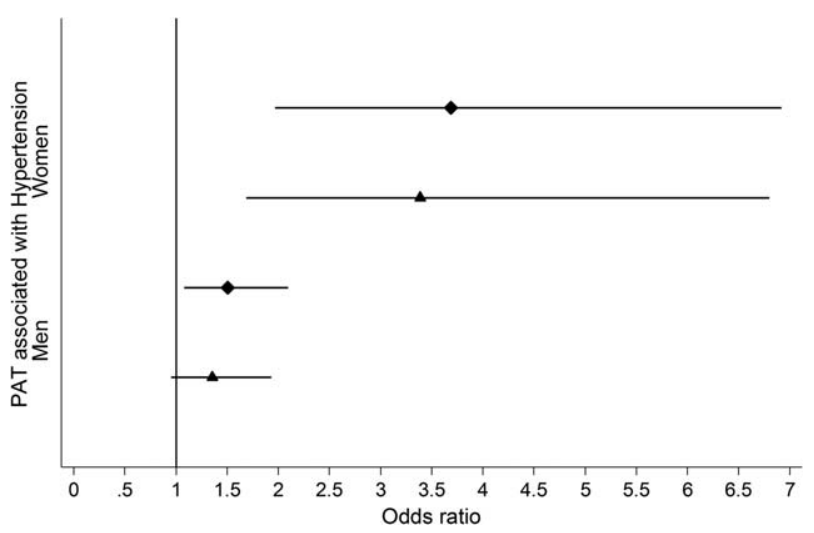

FIGURE 2 Association between pericardial adipose tissue and hypertension in men and women providing age-adjusted odds ratios (diamond) and fully adjusted odds ratios (triangle) with $95 \%$ confidence intervals.
WHtR displayed the strongest associations with hypertension of all anthropometric markers in the multivariableadjusted model. This observation is supported by the published literature, where several studies reported that markers of central adiposity including waist circumference, WHtR and WHR predict cardiovascular disease risk and hypertension better than BMI [19-22]. However, the present study also evaluated the associations of imaging-based direct measurements of body fat distribution with prevalent hypertension.

\section{Body and organ fat measurements and hypertension}

The associations of different CT-based fat measurements, including pericardial fat, intrathoracic fat and VAT with cardiovascular disease risk factors were evaluated in a sub-sample of the Framingham Offspring cohort ( $n=1155$ participants). One main result was, that VAT was more strongly associated with SBP, DBP and hypertension than pericardial (defined as adipose tissue located within the pericardium) and intrathoracic fat [23]; and that intrathoracic fat was more strongly associated with BP and hypertension than pericardial fat. Similarly, in our MRI study, VAT was more strongly associated with prevalent hypertension than PAT (as the sum of epicardial and paracardial fat; $\mathrm{OR}=1.85$ for VAT; $\mathrm{OR}=1.66$ for PAT, respectively). However, MR-derived measurements of TAT and SCAT displayed even higher ORs for hypertension as compared with VAT and PAT. Recently, we have investigated in detail the association between liver fat and blood pressure in the present sample and observed that three different HFF measurements (measured at three different locations within the liver) displayed consistent associations with $\mathrm{BP}$ and hypertension [24]; independently of the measurement location.

\section{Comparison between MRI-derived fat measurements and anthropometric markers with respect to their association with hypertension}

A comprehensive comparison of anthropometric markers and more direct fat measures determined by BIA, including total body fat, percentage body fat, trunk fat mass and percentage trunk fat, with respect to their association with hypertension was conducted in a sample from the Chinese general population and revealed female WHtR and male

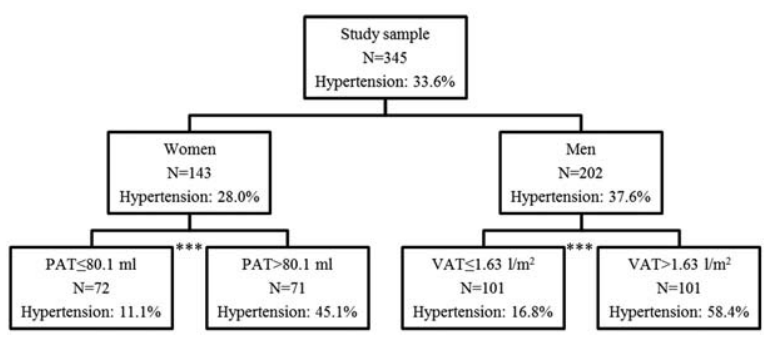

FIGURE 3 Classification tree of interaction analysis including all MRI and anthropometric markers (sex-specific median-dichotomized) for identifying hypertension according to chi-square automatic interaction detection adjusted for age, ${ }^{*} P<0.05,{ }^{* * *} P<0.001$. CHAID, chi-square automatic interaction detection. 
BMI (OR $=4.90 ; 95 \%$ CI 3.36-7.17; top vs. bottom quartile) as the strongest correlates for hypertension. In men, BMI displayed approximately a twofold OR for hypertension than percentage body fat (OR $=2.42 ; 95 \%$ CI $1.53-3.81)$. However, this difference in the strength of the association with prevalent hypertension was not apparent in women $\left(\mathrm{OR}_{\mathrm{BMI}}=3.92 ; 95 \%\right.$ CI $2.94-5.23$ vs. OR $\%$ BodyFat $=3.60 ; 95 \%$ CI 2.94-5.23, respectively). By contrast, in our study, we could not detect effect modifications by sex except for PAT, and the effect measure (OR per SD increment) for TAT was similar to the effect measure for BMI for both, men and women $\left(\mathrm{OR}_{\mathrm{TAT}}=1.97 ; \mathrm{OR}_{\mathrm{BMI}}=1.78\right)$. Although AUC values (for differentiating between individuals with vs. without hypertension) differed for individual fat measures, these differences disappeared after adding traditional risk factors (age, sex, diabetes mellitus, physical activity, smoking status, alcohol consumption, total cholesterol and HDLcholesterol) to the statistical model.

The study of Rosito et al. investigated whether intrathoracic or pericardial fat were associated with blood pressure and hypertension, independent of BMI and waist circumference or of VAT and observed that only intrathoracic fat displayed a borderline significant association with hypertension whenever added to a model including BMI and waist circumference in women and VAT, but not intrathoracic or pericardial fat, was independently and statistically significantly associated with SBP and hypertension only in women in multivariable models including both measurements (VAT and intrathoracic or pericardial fat, respectively) [23]. In our analyses, we detected that only TAT was associated with hypertension independently of basic cardiovascular risk factors in a statistical model that included the most strongly associated anthropometric marker, waist circumference.

\section{Effect modification by sex}

Our analyses revealed a strong and independent positive association of PAT with hypertension only in women but not in men. Furthermore, when all adiposity traits were dichotomized at the median, the low-PAT vs. highPAT groups displayed the greatest difference in hypertension prevalence (11 and 45\%, respectively) in women. In men, the low-VAT vs. high-VAT groups had the biggest difference in hypertension prevalence (17 and $58 \%$, respectively). Thus, based on dichotomization at the sex-specific median, MR-derived PAT and VAT could improve the prediction of hypertension and the associated cardiovascular risk in women and men, respectively.

Consistent with our observations, PAT was significantly associated with SBP and DBP only in women in the MESA (Multi-Ethnic Study of Atherosclerosis) study [25]. In contrast to our study, a stronger role of BMI in predicting risk for hypertension in women as compared with men has been reported in the study of Sakurai et al. [26] but not in the study of Zhang et al. [7].

Sex differences of associations between cardiovascular risk factors and cardiovascular diseases in women compared with men have been explained, for example, by cardioprotective effects of estrogen before and after menopause [27]. However, evidence for sex differences in the associations of fat distribution markers with hypertension is limited so far [7]. In our sample, no other associations of adiposity traits with hypertension were modified by sex.

\section{Strengths and limitations}

Strengths of our study are the well characterized subsample of the population-based KORA study, a cohort study with detailed and highly standardized cardiovascular phenotyping, and the use of advanced MR techniques to characterize body and organ fat content.

Our study is limited by its cross-sectional design so that we could not assess the association of fat distribution measures with new-onset (incident) hypertension. Although BP measurements were obtained of seated participants after a minimum resting period of $5 \mathrm{~min}, \mathrm{BP}$ elevation because of the presence of the BP-measuring personnel (white-coat hypertension) cannot entirely be excluded. The representativeness of the study sample for the initial cohort sample and the population of the study region is also limited. Reasons for nonresponse included contraindications for MRI examinations and refusal of informed consent and refusal of telephone invitation. Our sample was of European descent. The applicability of our findings to other ethnicities remains to be established. A comparison of MRI participants and non-MRI participants of the KORA FF4 cohort revealed that participants of the MRI sub-study were a bit younger, more often men and less often hypertensive compared with non-MRI participants (Supplemental Table 5, http://links.lww.com/ HJH/A923).

In conclusion, MRI-derived adipose tissue measurements perform similarly in identifying patients with hypertension compared with anthropometric markers. Especially, the MRI markers, TAT, VAT and PAT, were highly correlated with prevalent hypertension. Furthermore, the established anthropometric markers, waist circumference and WHtR, were also confirmed to be significantly and independently associated with hypertension. The longitudinal predictive performance of individual MRI fat measures and anthropometric markers with respect to changes in BP over time and incident hypertension needs to be investigated in future studies.

\section{AGKNOWLEDGEMENTS}

The contributions to data collection made by field workers, radiologists, technicians, interviewers and computer assistants are gratefully acknowledged.

Sources of funding: The KORA study was initiated and financed by the Helmholtz Zentrum München - German Research Center for Environmental Health, which is funded by the German Federal Ministry of Education and Research (BMBF) and by the State of Bavaria. Furthermore, KORA research was supported within the Munich Center of Health Sciences (MC-Health), Ludwig-Maximilians-Universität, as part of LMUinnovativ. The KORA-MRI sub-study received funding by the German Research Foundation (DFG, Deutsche Forschungsgemeinschaft) and the Centre for Diabetes Research (DZD e.V., Neuherberg, Germany). The KORA-MRI sub-study was supported by an unrestricted research grant from Siemens Healthcare. 


\section{Conflicts of interest}

There are no conflicts of interest.

\section{REFERENGES}

1. Mancia G, Fagard R, Narkiewicz K, Redon J, Zanchetti A, Bohm M, et al $2013 \mathrm{ESH} / \mathrm{ESC}$ Guidelines for the management of arterial hypertension: the Task Force for the management of arterial hypertension of the European Society of Hypertension (ESH) and of the European Society of Cardiology (ESC). J Hypertens 2013; 31:1281-1357.

2. Liu A, Abbasi F, Reaven GM. Adiposity indices in the prediction of metabolic abnormalities associated with cardiovascular disease in nondiabetic adults. Nutr Metab Cardiovasc Dis 2011; 21:553-560.

3. Hagg S, Fall T, Ploner A, Magi R, Fischer K, Draisma HH, et al. European Network for Genetic and Genomic Epidemiology Consortium. Adiposity as a cause of cardiovascular disease: a Mendelian randomization study. Int J Epidemiol 2015; 44:578-586.

4. Moliner-Urdiales D, Artero EG, Sui X, Espana-Romero V, Lee D, Blair SN. Body adiposity index and incident hypertension: the Aerobics Center Longitudinal Study. Nutr Metab Cardiovasc Dis 2014; 24 : 969-975.

5. Redon J, Tellez-Plaza M, Orozco-Beltran D, Gil-Guillen V, Pita Fernandez S, Navarro-Perez J, et al., ESCARVAL Study Group. Impact of hypertension on mortality and cardiovascular disease burden in patients with cardiovascular risk factors from a general practice setting: the ESCARVAL-risk study. J Hypertens 2016; 34:1075-1083.

6. D'Agostino RB Sr, Vasan RS, Pencina MJ, Wolf PA, Cobain M, Massaro JM, Kannel WB. General cardiovascular risk profile for use in primary care: the Framingham Heart Study. Circulation 2008; 117: $743-753$.

7. Zhang ZQ, Deng J, He LP, Ling WH, Su YX, Chen YM. Comparison of various anthropometric and body fat indices in identifying cardiometabolic disturbances in Chinese men and women. PLoS One 2013; 8:e70893.

8. Bamberg F, Hetterich H, Rospleszcz S, Lorbeer R, Auweter SD, Schlett CL, et al. Subclinical disease burden as assessed by whole-body MRI in subjects with prediabetes, subjects with diabetes, and normal control subjects from the general population: the KORA-MRI Study. Diabetes 2017; 66:158-169.

9. Zhong X, Nickel MD, Kannengiesser SA, Dale BM, Kiefer B, Bashir MR. Liver fat quantification using a multistep adaptive fitting approach with multiecho GRE imaging. Magn Reson Med 2014; 72:1353-1365.

10. Bertaso AG, Bertol D, Duncan BB, Foppa M. Epicardial fat: definition, measurements and systematic review of main outcomes. Arq Bras Cardiol 2013; 101:e18-e28.

11. Meisinger C, Heier M, Volzke H, Lowel H, Mitusch R, Hense HW, Ludemann J. Regional disparities of hypertension prevalence and management within Germany. J Hypertens 2006; 24:293-299.

12. Whitworth JA, World Health Organization ISoHWG. 2003 World Health Organization (WHO)/International Society of Hypertension (ISH) statement on management of hypertension. J Hypertens 2003; 21: 1983-1992.
13. Empfehlungen zur Hochdruckbehandlung in der Praxis und zur Therapie hypertensiver Notfälle. Heidelberg: Deutschen Liga zur Bekämpfung des hohen Blutdruckes e. V; 2010.

14. Meisinger C, Lowel H, Thorand B, Doring A. Leisure time physical activity and the risk of type 2 diabetes in men and women from the general population. The MONICA/KORA Augsburg Cohort Study. Diabetologia 2005; 48:27-34.

15. Keil U, Chambless LE, Doring A, Filipiak B, Stieber J. The relation of alcohol intake to coronary heart disease and all-cause mortality in a beer-drinking population. Epidemiology 1997; 8:150-156.

16. NCD Risk Factor Collaboration (NCD-RisC). Effects of diabetes definition on global surveillance of diabetes prevalence and diagnosis: a pooled analysis of 96 population-based studies with 331,288 participants. Lancet Diabetes Endocrinol 2015; 3:624-637.

17. Seissler J, Feghelm N, Then C, Meisinger C, Herder C, Koenig W, et al. Vasoregulatory peptides pro-endothelin-1 and pro-adrenomedullin are associated with metabolic syndrome in the population-based KORA F4 study. Eur J Endocrinol 2012; 167:847-853.

18. Carlsson AC, Riserus U, Engstrom G, Arnlov J, Melander O, Leander K, et al. Novel and established anthropometric measures and the prediction of incident cardiovascular disease: a cohort study. Int $J$ Obes (Lond) 2013; 37:1579-1585.

19. Lee JW, Lim NK, Baek TH, Park SH, Park HY. Anthropometric indices as predictors of hypertension among men and women aged 40-69 years in the Korean population: the Korean Genome and Epidemiology Study. BMC Public Health 2015; 15:140.

20. Janssen I, Katzmarzyk PT, Ross R. Waist circumference and not body mass index explains obesity-related health risk. Am J Clin Nutr 2004; 79:379-384

21. Lee HJ, Hwang SY, Hong HC, Ryu JY, Seo JA, Kim SG, et al. Waist-tohip ratio is better at predicting subclinical atherosclerosis than body mass index and waist circumference in postmenopausal women. Maturitas 2015; 80:323-328.

22. Ashwell M, Gibson S. Waist-to-height ratio as an indicator of 'early health risk': simpler and more predictive than using a 'matrix' based on BMI and waist circumference. BMJ Open 2016; 6:e010159.

23. Rosito GA, Massaro JM, Hoffmann U, Ruberg FL, Mahabadi AA, Vasan RS, et al. Pericardial fat, visceral abdominal fat, cardiovascular disease risk factors, and vascular calcification in a community-based sample: the Framingham Heart Study. Circulation 2008; 117:605-613.

24. Lorbeer R, Bayerl C, Auweter S, Rospleszcz S, Lieb W, Meisinger C, et al. Association between MRI-derived hepatic fat fraction and blood pressure in participants without history of cardiovascular disease. J Hypertens 2017; 35:737-744.

25. Brinkley TE, Jerosch-Herold M, Folsom AR, Carr JJ, Hundley WG, Allison MA, et al. Pericardial fat and myocardial perfusion in asymptomatic adults from the Multi-Ethnic Study of Atherosclerosis. PLoS One 2011; 6:e28410.

26. Sakurai M, Miura K, Takamura T, Ota T, Ishizaki M, Morikawa Y, et al. Gender differences in the association between anthropometric indices of obesity and blood pressure in Japanese. Hypertens Res 2006; 29:75-80.

27. Wells GL. Cardiovascular risk factors: does sex matter? Curr Vasc Pharmacol 2016; 14:452-457. 